\title{
USO DA SOMATOTROPINA BOVINA RECOMBINANTE (rbST) EM VACAS MESTIÇAS: UMA REVISÃO
}

Coralline Barbosa da Silva ${ }^{1}$ José Reinaldo Mendes Ruas²

SILVA, C. B. da; RUAS, J. R. M. Uso da somatotropina bovina recombinante (rbST) em vacas mestiças: uma revisão. Arquivos de Ciências Veterinárias e Zoologia da UNIPAR, Umuarama, v. 23, n. 2cont., e2303, 2020.

RESUMO: A maioria dos estudos sobre o uso da somatotropina bovina recombinante (rbST) foram conduzidos em países de clima temperado utilizando animais de genética Bos taurus e todo o protocolo de utilização foi pautado para tais animais e extrapolados aos mestiços (Bos taurus x Bos indicus) em ambientes tropicais. No entanto, existem características diferenciadas da curva de produção de leite e alguns aspectos fisiológicos que diferem os mestiços dos taurinos, sendo assim, estabelecer padrões específicos para uso do rbST para vacas mestiças podem melhorar a eficiência, reduzir custos e expandir o uso da tecnologia para os sistemas brasileiros. Estabelecer ajuste da dosagem, o intervalo, o melhor tempo para início e término da aplicação, avaliar o melhor retorno financeiro do uso para o sistema produtivo assim como as respostas produtivas e reprodutivas das vacas pode trazer adequação do uso da tecnologia no sistema de produção de gado mestiço leiteiro. $\mathrm{O}$ objetivo da revisão é identificar critérios a serem considerados para uso do rbST em vacas mestiças a fim de potencializar a estratégia do uso do mesmo. O rbST promove notório aumento da produção de leite e mais detalhes do protocolo de uso do hormônio para vacas mestiças necessita ser avaliado já que algumas características da curva de leite e capacidade produtiva são diferentes para animais cruzados.

PALAVRAS-CHAVE: Cruzamentos. Hormônio do crescimento. Produção de leite. Sistema de produção F1. Eficiência financeira.

\section{USE OF RECOMBINANT BOVINE SOMATOTROPIN (rbST) IN CROSSBRED COWS: REVIEW}

ABSTRACT: Most studies on the use of recombinant bovine somatotropin (rbST) were conducted in countries with temperate climates using Bos taurus animals and the entire use protocol was based on such animals and extrapolated to crossbred animals (Bos taurus x Bos indicus) in tropical environments. However, there are different characteristics in the milk production curve and some physiological aspects that differentiate the crossbred from those cattle. Therefore, the establishment of specific standards for the use of rbST for crossbred cattle can improve efficiency, reduce costs, and expand the use of technology to Brazilian systems. Establishing dosage adjustment, the interval, the best time to start and end the application, evaluating the best financial return from use on the productive system, as well as the productive and reproductive responses of the cows can help foster the adequacy of the use of technology in the production system of crossbred dairy cattle. The purpose of this review is to identify criteria to be considered for the use of rbST in crossbred cows in order to enhance the strategy of using it. The use of rbST promotes a noticeable increase in milk production; however, further details of the hormone use protocol for crossbred cows need to be evaluated since some characteristics of the milk curve are different for crossbred animals.

KEYWORDS: Crossings. Growing hormone. Milk production. F1 production system. Financial efficiency.

\section{USO DE SOMATOTROPINA BOVINA RECOMBINANTE (rbST) EN VACAS MESTIZAS: UNA REVISIÓN}

RESUMEN: La mayoría de los estudios sobre el uso de somatotropina bovina recombinante (rbST) se han realizado en países de clima templado utilizando animales de genética Bos taurus y todo el protocolo de utilización se ha pautado para tales animales y extrapolados a los mestizos (Bos taurus x Bos indicus) en ambientes tropicales. Sin embargo, existen diferentes características de la curva de producción de leche y algunos aspectos fisiológicos que diferencian al mestizo de los toros, por lo tanto, establecer estándares específicos para el uso de rbST para vacas mestizas puede mejorar la eficiencia, reducir costos y expandir el uso de tecnología para Sistemas brasileños. Establecer el ajuste de dosis, el intervalo, el mejor momento para iniciar y finalizar la aplicación, evaluar el mejor retorno económico del uso para el sistema productivo, así como las respuestas productivas y reproductivas de las vacas pueden propiciar la adecuación del uso de la tecnología en el sistema de producción de ganado mestizo lechero. El propósito de la revisión es identificar los criterios que se deben considerar para el uso de rbST en vacas mestizas con el fin de mejorar la estrategia de uso. El rbST promueve un aumento notable en la producción de leche y es necesario evaluar más detalles del protocolo de uso de hormonas para vacas cruzadas, ya que algunas características de la curva de la leche son diferentes para los animales cruzados.

PALABRAS CLAVE: Cruces. Hormona del crecimiento. Producción de leche. Sistema de producción F1. Eficiencia financiera.

DOI: 10.25110 /arqvet.v23i2cont.2020.6650

${ }^{1}$ Mestre em Zootecnia pela Universidade Estadual de Montes Claros - Unimontes. Av. Reinaldo Viana, 2630, Janaúba, MG. coralline15@hotmail.com ${ }^{2}$ Professor da Universidade Estadual de Montes Claros - Unimontes - Departamento de Ciências Agrárias. jrmruas@gmail.com 


\section{Introdução}

O aumento da produção de leite pode ocorrer por melhorias do manejo geral, pelo incremento genético (animais mais especializados) e adoção de tecnologias por meio da definição de estratégias condizentes com o sistema produtivo, sendo a somatotropina bovina recombinante uma alternativa ao sistema para melhoria da produção de leite. O principal efeito da Somatotropina bovina recombinante (rbST) é o aumento da produção de leite por meio de modificações no metabolismo de carboidratos, lipídeos e proteínas promovendo alterações da partição de nutrientes entre os tecidos e aumentando a síntese do leite da vaca (Bauman, 1992). No entanto, a maioria dos estudos com o hormônio foi conduzida em países de clima temperado utilizando animais de genética Bos taurus e todo o protocolo de utilização foi pautado para tais animais e extrapolado para vacas mestiças (B. taurus x B. indicus) em ambientes tropicais (COELHO et al., 2012). O BSTr é uma tecnologia importante para criadores de bovinos leiteiros pois impacta positivamente na lucratividade (MORAIS et al., 2017).

Entre 1975 a 1992 a Empresa Brasileira de Pesquisa Agropecuária conduziu diversos trabalhos com o intuito de determinar estratégias de acasalamentos e definir o grau de sangue mais adequado para as condições de sistema produtivo brasileiro e promoveu teste de progênie de touros mestiços (VERNEQUE et al., 2016) o que contribuiu para aumento da produção de leite e estima-se que mais de $74 \%$ do rebanho brasileiro é constituído por animais mestiços (VILELA, 2003), que diferem no comportamento da curva de lactação: momento do pico, persistência à lactação, comprimento da lactação e mobilização de reservas corporais, sendo assim estabelecer padrões específicos para uso do rbST podem melhorar a eficiência, reduzir custos e expandir o uso da tecnologia para os sistemas brasileiros. Além de avaliar o incremento produtivo, torna-se necessário identificar as melhores formas de uso do hormônio avaliando retorno financeiro com o uso do mesmo.

Respostas técnicas-financeiras satisfatórias com o uso do rbST são conseguidas por meio de ajustes dos manejos sanitário e principalmente nutricional das vacas no sistema produtivo, podendo assim potencializar a resposta dos animais com o hormônio quando os mesmos estão em boas condições de manejo geral.

Dessa forma, o objetivo dessa revisão é identificar alguns pontos para melhor adequação da utilização do rbST em animais mestiços, destacando os critérios de uso, características das vacas mestiças, efeitos da estratégia adotada, efeitos produtivos, reprodutivos e financeiros com a utilização desse hormônio.

\section{Desenvolvimento}

\section{Somatotropina Bovina Recombinante (rbST)}

A ST bovina (bST) pode ter 190 ou 191 aminoácidos na sua cadeia peptídica podendo diferir o aminoácido da posição 126 (podendo ser valina ou leucina), sendo esta sequência pouco semelhante à ST humana (BAUMAN, 1992; ETHERTON; BAUMAN, 1998). É considerado um hormônio com receptores específicos a cada espécie, mas, em alguns casos, pode haver grandes semelhanças na cadeia peptídica que promovem certa ação biológica em espécies diferentes - sendo considerado "espécie limitado" (COELHO et al., 2013) desde que hajam receptores fisiológicos de ligação.

Com o advento da engenharia genética iniciou-se a produção do bST a partir da técnica do DNA recombinante, aperfeiçoada e utilizada até hoje, ampliando sua produção em larga escala (BAUMAN, 1992; ETHERTON; BAUMAN,1998; BAUMAN; COLLIER, 2010). Após diversos estudos e comprovações sobre o impacto desse hormônio tanto para saúde animal quanto humana e seu efeito na produção, em 1994, a US Food an Drugs Administration (FDA) liberou a rbST para uso comercial nos Estados Unidos e o mesmo ocorreu em outros países.

A ST exógena (rbST) deve ser administrada de forma injetável - como todo hormônio proteico (BAUMAN, 1992). No Brasil existem dois produtos comerciais do rbST de liberação contínua que diferem quanto ao veículo utilizado: a lecitina da soja e vitamina E (acetato) - SE-rbST e o outro tem como veículo o óleo de gergelim e zinco - GZrbST (CAMPOS, 2013; MORAIS et al., 2017), e a diferença no veículo parece promover uma diferença de velocidade na liberação da molécula de somatotropina, tendo o primeiro produto liberação mais rápida (ALMEIDA, VIECHNIESKI, 2011), enquanto o segundo produto comercial promoveu maior produção de leite $(+2,03 \mathrm{~kg} / \mathrm{d}$ paraa primíparas e $+1,32$ $\mathrm{kg} / \mathrm{d}$ para multíparas, respectivamente para GZ-rbST e SE$\mathrm{rbST})$, maior rendimento de gordura $(+70 \mathrm{~g} / \mathrm{d}$ para primíparas e $+40 \mathrm{~g} / \mathrm{d}$ para multíparas, $P<0,01)$, maior rendimento de proteína no leite nas primíparas $(+70 \mathrm{~g} / \mathrm{d} P<0,01)$ e melhor persistência lactacional $(-0,65$ vs. $-0,73, P<0,01)$ ao longo do ciclo lactação completo das vacas decorrente da menor variação da resposta dentro do ciclo de aplicação do BSTr (MORAIS et al., 2017).

Ambos possuem recomendações de uso similares em que se inicia a aplicação na nona semana após o parto, via subcutânea e a intervalos de 14 dias (LUCCI et al., 1998).

\section{Mecanismo de ação}

A ST é um hormônio de crescimento sintetizado pela glândula pituitária sendo de origem proteica e transportada pelo sangue para ação em diversos tecidos (BAUMAN, 1992; ETHERTON, BAUMAN, 1998). Sua secreção pode ser estimulada por meio do fator de liberação da somatotropina (GRF) ou pode ser inibida a liberação pela somatostatina (ETHERTON, BAUMAN, 1998).

A ST bovina é considerada um hormônio homeorrético, promove uma redução da utilização dos nutrientes pelos tecidos periféricos. A glândula mamária possui receptores do IGF-I promovendo o aumento do fluxo sanguíneo e influenciando o encaminhamento dos nutrientes ingeridos para a mesma onde há aumento da síntese do leite, sendo todo o processo fisiológico determinado por modificações da resposta no metabolismo de carboidratos, proteínas, lipídeos e minerais nos animais (ETHERTON; BAUMAN,1998) e consequentemente maior resposta galactopoietica (CHALUPA; GALLIGAN, 1989).

Em animais tratados com rbST as taxas de gliconeogênese no fígado se elevam, sendo que para animais 
ruminantes grande parte da glicose produzida é oriunda desse metabolismo por meio do propionato (ácido graxo volátil absorvido no rúmen), sendo de até 2,3 vezes maior a glicose produzida por gliconeogênese em animais tratados com BST (KNAPP et al., 1992). Além desse aumento há uma influência do GH minimizando a utilização dessa glicose pelos músculos e outros órgãos, aumento do fluxo sanguíneo e maior captação dos nutrientes pela glândula mamária (CHEREPANOV, DANFAER, CANT, 2000). Bauman (1999) afirma haver uma redução da insulina na inibição da gliconeogênese.

O bST estimula a produção de IGF-I pelos adipócitos, mas os mesmos não possuem receptores (BAUMAN, 1998). Segundo Lucy et al. (2001) o bST promove aumento da lipólise e consequente aumento dos ácidos graxos não esterificados na circulação (AGNE) que podem ser utilizados na síntese do leite ou oxidados no fígado, além disso, promove um bloqueio da lipogênese dependente da insulina que é reduzida pelo aumento do bST circulante.

Bauman (1998) cita que o metabolismo lipídico com o uso do bST em animais em balanço energético negativo acarreta um aumento no uso das reservas corporais. Em balanço positivo (com regulação do consumo) há um reajuste do metabolismo lipídico e restabelecimento das reservas, havendo um feito apenas inibitório na lipogênese e uma modificação da lipólise, teor de gordura e composição de ácidos graxos do leite (BAUMAN; VERNON, 1993), no entanto, o consumo de alimento com o uso do bST em vacas é mais demorado (em torno da oitava semana) e pode então promover um pequeno estado de balanço negativo nos animais.

Não é muito claro o efeito do bST sobre o metabolismo proteico (ETHERTON; BAUMAN, 1998), mas sabe-se que há aumento da síntese e ganho de proteína muscular em animais em crescimento (SCHLEGEL et al., 2006) e aumento da síntese de sólidos no leite em animais em lactação (ETHERTON, BAUMAN, 1998).

Todas essas modificações fisiológicas promovem aumentos na produção de leite e podem ou não afetar a condição corporal do animal e promover efeitos na reprodução, e por esses fatores a estratégia de uso rbST deve obedecer aos critérios para melhores respostas nas vacas.

\section{Efeito na produção e composição do leite}

É notoriamente bem definido o efeito do bST sobre o aumento da produção de leite (BAUMAN, 1992; BAUMAN E ETHERTON, 1998). Diversos trabalhos têm demonstrado o efeito desse hormônio tanto para vacas holandesas (CAPPER et al., 2008; RENNÓ et al., 2006; SANTOS et al., 2001) quanto para cruzados (CAMPOS et al., 2011; FONTES et al. 1997; OLIVEIRA NETO et al. 2001). Existe um efeito direto de modificação da curva de produção com aumento imediato da produção de leite e persistência, e as respostas são bastante dependentes das doses utilizadas do rbST (CAMPOS et al., 2011; RENNÓ et al., 2006; SANTOS et al., 2001;) além de diversos fatores que também influenciam a produção de leite, como: ordem de lactação, forma de administração, qualidade e adaptação de exigências da dieta, estádio de lactação e manejo geral (MATTOS et al., 1990), assim como o status nutricional, condição corporal da vaca e a variação individual existente na resposta (COELHO et al., 2010), devendo os mesmos serem considerados pois podem influenciar a resposta de ação do bST (BAUMAN, 1992). Em condições de manejo precárias (manejo sanitário e/ou nutricional deficiente, vacas estressadas) não há resposta ao uso do rbST, e a resposta melhora a medida que há incremento no manejo geral (BAUMAN, 1992). Sendo assim para indicação do uso do hormônio nos sistemas leiteiros é necessário avaliar se é condizente o desembolso com o hormônio, pois a resposta dependerá do patamar de preparo do manejo básico - sanitário e nutricional da propriedade.

Avaliações quanto à diferentes dosagens e influência na composição do leite foram realizadas por Santos et al. (2001) em vacas holandesas onde houve aumento da produção de leite em $1,4,4,1$ e $0,8 \mathrm{~kg} / \mathrm{vaca} /$ dia respectivamente nas doses 250,350 e $500 \mathrm{mg}$, não havendo diferenças entre os tratamentos quanto à porcentagem de sólidos do leite mas notou-se um aumento da gordura no tratamento com 350mg de rbST e relativa redução da proteína total do leite (mas não foi suficiente para afetar o seu valor nutritivo).

Fontes et al. (1997) encontraram aumento médio de $2,5 \mathrm{~kg} / \mathrm{dia}$ e $2,6 \mathrm{~kg} / \mathrm{dia}$ (sem diferença estatística) para dose de $250 \mathrm{mg}$ e $500 \mathrm{mg}$ de rbST em animais mestiços comparado ao placebo.

Oliveira Neto et al. (2001) observaram aumento médio de 2,4 kg leite/dia em vacas 1/2 e 3/4 Holandês-Zebu (HZ) tratadas com o rbST, e aumento similar médio foi obtido por Campos et al. (2011) sendo de 2,3kg/dia em vacas mestiços $3 / 4$ e $7 / 8 \mathrm{HZ}$, ambos os trabalhos utilizando dose de $500 \mathrm{mg}$.

Rennó et al. (2006) observaram aumento de 14\% e $13,2 \%$ respectivamente para produção de leite e produção de leite corrigida para gordura em vacas holandesas (administradas doses de 500mg iniciada aos 60 dias) sem alterar o desempenho reprodutivo das vacas. Bauman (1992) afirma não haver modificações na composição bruta do leite de animais tratados com rbST, porém, até que o animal ajuste seu metabolismo ao tratamento pode haver um certo aumento da gordura do leite principalmente nas primeiras semanas da administração do hormônio.

Rennó et al (2006) encontraram menor queda da produção de leite após o pico (maior persistência), para holandês, em tratamentos iniciados aos 60 dias ao invés de 100 dias - respectivamente com quedas de $25,7 \mathrm{~g} /$ dia de leite por dia e 55,5g de leite/dia em relação ao controle.

\section{Efeito do rbST na reprodução}

Lucy (2000) afirma não haver efeito direto do rbST sobre a reprodução, mas sim efeito pelo aumento da produção de leite e do balanço energético negativo pós-parto que podem impactar o escore de condição corporal (BAUMAN et al., 1985; BAUMAN; VERNON, 1993).

Lucy (2001) defende que o efeito do bST na reprodução é dependente da visão técnico-estratégica para avaliação do estado nutricional e alimentar dos animais. Lucy (2000) e Rennó et al. (2006) observaram aumentos produtivos sem haver redução do desempenho reprodutivo das vacas. Da mesma forma, Campos et al. (2011) observou além do aumento do leite produzido a interação entre dose 
e início da aplicação em vacas mestiças para características reprodutivas: considerando dias em aberto, a dose de $250 \mathrm{mg}$ aplicada 40 dias pós-parto obteve melhor resposta que a dose de $500 \mathrm{mg}$, quando aplicada aos 60 dias pós-parto a dose de $500 \mathrm{mg}$ obteve melhor resposta comparada a $250 \mathrm{mg}$, o mesmo efeito ocorreu para número de serviços por prenhes, mas não houve diferenças quando avaliado o intervalo de parto por prenhes. É importante destacar que as características reprodutivas sofrem grande impacto de diversos fatores ambientais.

Com avanço das biotecnologias aplicadas à reprodução, como a transferência de embriões, tem se afirmado a importância da quantidade e qualidade dos oócitos produzidos. Baruselli et al., (2020) em revisão de literatura destaca o uso do rbST com o intuito de aumentar o número de folículos ovarianos na superestimulação ovariana das vacas possivelmente pelo aumento do número de receptores para IGF-1. Ribeiro et al. (2020) não observaram diferenças na quantidade de folículos ovarianos quando testada dose de 250 e $500 \mathrm{mg}$ de rbST para vacas da raça Sindi, mas a maior dose promoveu maior número de oócitos viáveis (91,52\% vs. $53,33 \%$ ) e independente da dose o uso do rbST promoveu maior número de folículos grandes $(0,42$ vs. $0,0 \quad P<0,05$ comparada ao controle).

Nutrição e rbST

Coelho et al. (2012) citam que o efeito do rbST está associado ao balanço energético das vacas pois a produção de IGF-I é dependente do número de receptores do hormônio no fígado e este número se modifica no balanço energético negativo e/ou em animais mal alimentados, e seu uso deve ser destinado a animais com boas condições corporais (indicado escore mínimo de 3,00).

Animais com balanço energético positivo possuem em contrapartida melhor resposta ao rbST pois possuem receptores ativos do hormônio no fígado, o que aumenta o IGF-I e consequentemente o efeito na síntese do leite (AKERS, 2002). Coelho et al. (2010) admitem que o comportamento de resposta em animais mestiços, tendo pico de lactação mais precoce apresentam, possivelmente, menores efeitos do balanço energético negativo, retornando mais cedo o direcionamento dos nutrientes para reserva corporal ao invés da produção de leite, e considerando esta peculiaridade em vacas mestiças, especula que o uso do rbST pode ser feito mais precocemente em vacas mestiças.

Com o uso do rbST há a modificação da curva de produção de leite, obtendo aumento imediato na produção produção vertical - e uma minimização da queda de produção após o pico (CHALUPA; GALLIGAN, 1989).

O uso do rbST não promove mudança nas exigências de mantença (BAUMAN, 1999) e não há a necessidade de utilização de dietas especiais sendo a mesma dieta usada para animais em lactação usada também para vacas lactantes tratadas com rbST, porém é importante que os a mesma supra as exigências das vacas (necessidades nutricionais para mantença, produção de leite, gestação e reposição de reservas corporais) (COELHO et al. 2012).

Bauman (1992) cita que, após algumas semanas, há um ajuste considerável do consumo alimentar nas vacas tratadas com o bST, o qual depende da taxa de incremento produtivo. Atenção especial deve ser dada aos fatores de manejo que afetam a ingestão de alimentos e a qualidade da forragem é tido como ponto crítico (COELHO et al. 2012).

\section{Caracterização da lactação e produção de leite em animais mestiços leiteiros}

Para Glória et al. (2010) conhecer a curva de lactação auxilia na criação de estratégias de manejo sobre os animais podendo gerar respaldo com aparato técnico para descarte precoce, a avaliação de reprodutores e planejamento alimentar da atividade.

Há diferenciações de comportamento da curva de lactação das vacas mestiças em relação às holandesas, sendo observados picos mais discretos de produção de leite em animais F1 HZ (GLÓRIA et al., 2010) que devem ser consideradas no manejo geral e planos técnicos estratégicos (COBUCCI et al., 2000).

Em estudos sobre as curvas de lactação com animais oriundos de diversos cruzamentos genéticos, Glória et al. (2010) encontraram intervalo de lactação até o pico médio de 23 dias para animais cruzados.

Glória et al. (2012) encontraram diferenças desse período de acordo com a época do ano obtendo em média um intervalo de 30 dias. Vacas holandesas geralmente apresentam pico de produção entre 42 e 56 dias (COELHO et al., 2013). Vacas mestiças possuem curva de produção com a fase ascendente (do parto até o pico) menos pronunciada que animais europeus, caracterizada por Oliveira et al. (2007) como curva com tendência linear decrescente com média de 270 dias de duração da lactação.

Peixoto (2011) cita que a menor produção, duração da lactação e persistência em animais mestiços pode estar ligadas à regulação do eixo somatotrópico em que os mesmos direcionam os nutrientes ingeridos para crescimento em detrimento da produção de leite possivelmente pela existência de um perfil metabólico menos intenso e desacoplamento do eixo somatotrópico natural das vacas mestiças após o parto.

A resposta em produção para animais cruzados quanto à dose, início do tratamento e período de administração (em semanas) pode impactar diretamente os custos produtivos, visto que uma melhor dose resposta e o período em que o animal está responsivo em produção de leite ajudam a definir com mais clareza a adequação da estratégia baseada na melhor resposta técnica e financeira.

\section{Impacto financeiro da incorporação de tecnologias na atividade leiteira}

Sistemas que desembolsam com a compra de insumos ganham em escala de produção e maior chance de sobrevivência e expansão do negócio. Sistemas que possuem manejo geral adequado dos animais, podem intensificar a utilização das vacas e aumentar a produção com a adoção de estratégias, e o bST pode ser uma alternativa para tal, no entanto, avaliar a resposta financeira passa a ser um critério de suma importância para adoção.

Smith, Bailey e Hardin (1995) avaliando as implicações financeiras com gado holandês e o uso do rbST, observaram um pequeno aumento dos custos oriundo do ajuste de consumo da dieta e com o custo do produto, mas houve promoção de melhor retorno financeiro com a utilização do hormônio, que foi ainda maior quando houve 
aumento no número de ordenhas diárias - de duas para três ordenhas. Dessa forma, fica evidente que as estratégias em gado de leite podem ser somadas para potencializar o retorno na atividade. No entanto, para avaliar a melhor resposta técnica e financeira é primordial que se conheça e estabeleça protocolo de uso da estratégia para os genótipos e condições brasileiras (FONTES et al. 1997; COELHO et al. 2012; CAMPOS, 2013).

\section{Considerações Finais}

A utilização do rbST promove aumentos na produção de leite comprovada em diversos estudos por todo o mundo, porém a estratégia utilizada foi baseada em animais de sangue holandês e expandida para os demais grupos genéticos existentes na produção leiteira brasileira, sendo assim, maiores estudos devem ser considerados baseado no melhor custo/benefício, além disso o protocolo para animais mestiços em sistemas tropicais deve ser melhor determinado.

\section{Referências}

AKERS, R. M. Somatotropin. In: Lactation and the mammary gland. Iowa: Blackwell, 2002. cap.7, p.181195.

ALMEIDA, R.; VIECHNIESKI, S. L. Effect of shortterm treatment with bovine somatotropina on milk yield of Brazilian dairy cow. Journal of Dairy Science, v. 94 E-Suppl. 1, p. 355, (Abstr.), 2011.

BARUSELLI, P. S. et al. Estratégias para aumentar a produção de embriões em bovinos. In: Anais do XXIII Congresso Brasileiro de Reprodução Animal (CBRA2019); Gramado, RS, 2019.

BAUMAN, D. E. Bovine somatotropin and lactation: from basic science to commercial application. Domestic Animal Endocrinology, v.17, p.101-116, 1999.

BAUMAN, D. E. Bovine somatotropin: review of an emerging animal technology. Journal of Dairy Science, v. 75, p. 3433-3451, 1992.

BAUMAN, D. E.; VERNON, R. G. Effects of exogenous bovine somatotropin on lactation. Annual Review Nutrition, v. 13, p. 437-461, 1993.

BAUMAN, D. E.; COLLIER, J. R. Use of bovine somatotropin in dairy production. 2010. Disponível em: http://agribiotech.info/details/2010rBSTR Acesso: 05 mar. 2018.

CAMPOS, B. G. Somatotroprina bovina recombinante (bST): efeitos de dose, início de aplicação e intervalo de aplicação no desempenho produtivo de vacas mestiças Holandês-Gir. 2013. 116f .Tese ( Doutorado em Zootecnia) - Universidade Federal de Minas Gerais, 2013.

CAMPOS, B. G. et al. Emprego da somatotropina bovina (bST) de $500 \mathrm{mg}$ em vacas mestiças Bos taurus x Bos indicus a intervalos de 12 ou 14 dias. A Hora Veterinária, p. 8-13, 2011.

CAPPER, J. L. et al. The environmental impact of recombinant bovine somatotropin (rbSTr) use in dairy production. Proceedings of the National Academy of Sciences, v. 105, p. 9668-9673, 2008.

CHALUPA, W.; GALLIGAN, D. T. Nutricional implications of somatotropin for lactating cows. Journal of Dairy Sciences, v. 72, p. 2510-2524, 1989.

CHEREPANOV, G. G.; DANFAER, A.; CANT, J. P.

Simulation analysis of substrate utilization in the mammary gland of lactating cows. Journal of Dairy Research, v. 67, p. $171-188,2000$.

COBUCCI, J. A. et al. Curva de lactação na raça Guzerá. Revista Brasileira de Zootecnia, v. 29, n. 5, p. 1332-1339, 2000 .

COELHO, S. G. et al. Mecanismos de ação do bstr e uso em vacas mestiças. In: Anais IV Simpósio Mineiro de Nutrição de Gado de Leite - Belo Horizonte: FEPMVZ Editora, p. 190-201, 2012.

COELHO, S. G. et al. Mecanismos de ação do bSTr e uso em vacas mestiças. Revista VeZ em Minas, v. 116, n. 1, 2013.

COELHO, S. G. et al. Práticas de manejo para melhorar a eficiência produtiva de vacas mestiças F1 Holandês x Zebu. Informe Agropecuário. Belo Horizonte, v. 31, n. 258, p. 81-89, set./out. 2010.

ETHERTON, T. D.; BAUMAN, D. E. Biology of somatotropin in growth and lactation of domestic animals. Physiological Reviews, v. 78, p. 745-761, 1998.

FAEMG. Diagnóstico da pecuária leiteira no estado de Minas Gerais em 2005. Belo Horizonte: FAEMG, 156 p. 2006.

FDA. Food and Drug Administration. Bovine Somatotropin (BST). Disponível em: https://www.fda.gov/ animalveterinary/safetyhealth/productsafetyinformation/ ucm055435.htm. Acesso em: 09 maio 2017.

FONTES JR., C. et al. Response of brazilian crossbred cows to varying doses of bovine somatotropin. Journal of Dairy Sciences, v. 80, p. 3234-3240, 1997.

GLÓRIA, J. R. et al. Curvas de lactação de quatro grupos genéticos de mestiças Holandês-Zebu. Revista Brasileira de Zootecnia, v. 39, n. 10, p. 2160-2165, 2010.

GLÓRIA, J. R. et al. Enviromental and genetic effects on the lactation curves of four genetics groups of crossbred Holstein-zebu cow. Revista Brasileira de Zootecnia, v. 41 n. 11, p. 2309-2315, 2012. 
KNAPP, J. R. et al. Effects of somatotropin and substrates on patterns of liver metabolism in lactating dairy cattle. Journal of Dairy Science, v. 75, p. 1025-1035, 1992.

LUCCI, C. S. et al. Emprego da somatotropina bovina (BST) em vacas de alta produção. Brazilian Journal of Veterinary Research and Animal Science, São Paulo, v. 35, n. 1, p. 46-50, 1998.

LUCY, M. C. Regulation of ovarian follicular growth by somatotropin and insulinlike growth factors in cattle. Journal of Dairy Science, v. 83, p. 1635-1647, 2000.

LUCY, M. C.; JIANG, H.; KOBAYASHI, Y. Changes in the somatotropina axis associated with the initiation of lactation. Journal of Dairy Science, 84, e113-e119, 2001.

MATTOS, W. R. S. Somatotropina bovina e suas implicações nos processos de secreção de leite. In: MOURA, J. C.; FARIA, V. P.; PEIXOTO, A. M. (Org.). Novas Tecnologias de Produção Animal. Piracicaba: FEALQ, p. 71-86, 1990.

MORAIS, J. P. G. et al. Lactation performance of Holstein cows treated with 2 formulations of recombinant bovine somatotropin in a large commercial dairy herd in Brazil. Journal of Dairy Science, v. 100, p. 5945-5956, 2017.

OLIVEIRA NETO, J. B., MOURA, A. A. A.; NEIVA, J. N. M. Indicadores de estresse térmico e utilização da somatotropina bovina (bST) em vacas leiteiras, mestiças (Bos taurus x Bos indicus) no semi-árido do nordeste. Revista Brasileira de Zootecnia, v. 30, n. 2, p. 360-367, 2001.

OLIVEIRA, H. T. V. et al. Curvas de lactação de vacas F1 Holandês-Gir ajustadas pela função gama incompleta. Arquivo Brasileiro de Medicina Veterinária e Zootecnia, v. 59, p. 233-238, 2007.

PEIXOTO, R. T. Perfil metabólico e hormonal de vacas f1 Holandês-zebu durante o pré e pós-parto e sua relação com a reprodução. 2011. 45f. Dissertação (Mestrado em Zootecnia) - Escola de Veterinária, Universidade Federal de Minas Gerais, Belo Horizonte.

RENNÓ, F. P. et al. Efeito da somatotropina bovina recombinante (rBST) sobre o desempenho produtivo e reprodutivo de vacas da raça Holandesa. Arquivo

Brasileiro de Medicina Veterinária e Zootecnia, v. 58, n. 2, p. 158-166, 2006.

RIBEIRO, L. G. M. et al. Efeito da somatotropina bovina recombinante (rbST) na produção In vivo e na maturação In vitro de oócitos bovinos da raça Sindi. Arquivo Brasileiro de Medicina Veterinária e Zootecnia, v. 72, n. 1, p. 25-32, 2020 .

SANTOS, R. A. et al. Efeito de diferentes doses de somatotropina bovina (rBST) na produção e composição do leite. Ciência e Agrotecnologia, Lavras, v. 25, n. 6, p.
1435-1445, nov./dez. 2001.

SCHLEGEL, M. L. et al. Use of bovine somatotropin for increased skeletal and lean tissue growth of Holstein steers. Journal Animal Science, v. 84, p. 1176-1187, 2006.

SMITH, J.; BAILEY, K. W.; HARDIN, K. D. Financial implications of rBST. In: Westerb Large Herd Dairy Management Conference, 2, 1995, Las Vegas.

Proceedings... Las vegas, p.19-26, 1995.

VERNEQUE, R. S. et al. A contribuição do melhoramento animal para a pecuária de leite. In: VILELA, D. et al. Pecuária de leite no Brasil: cenários e avanços tecnológicos. Brasília, DF: Embrapa, p. 105-126, 2016.

VILELA, D. Cruzamento errado pode deteriorar genética. Noticiário Tortuga, São Paulo, ano 49, n.432, jul./ ago.2003.

Recebido em: 06.03.2018 Aceito em: 28.09.2020 